\title{
Disease and Diagnosis
}

\section{Menstrual Patterns and Disorders Among Adolescent Girls in Bandar Abbas, Iran}

\author{
Sajjad Shamloo ${ }^{1}$, Azin Alavi $^{\circledR}{ }^{\circledR}$, Koorosh Nematpour $^{3^{*}(}$, Zahra Mirshekari $^{1 \oplus}$, Abolfazl Taheri $^{1 \oplus}$ \\ ${ }^{1}$ Student Research Committee, Hormozgan University of Medical Sciences, Bandar Abbas, Iran. \\ ${ }^{2}$ Obstetrics and Gynecology Department, Khalij Fars Infertility Research Center, Hormozgan University of Medical \\ Sciences, Bandar Abbas, Iran. \\ ${ }^{3}$ Infectious and Tropical Diseases Research Center, Hormozgan University of Medical Sciences, Bandar Abbas, Iran.
}

\begin{abstract}
Background: Menstrual cycle is one of the important factors to protect women's sexual health, and it is affected by various factors in different individuals. Disrupting the balance of this cycle imposes numerous impacts on women's health. The present study aimed to investigate the incidence rate and factors associated with menstrual disorders among high school girls in Bandar Abbas, Iran.

Materials and Methods: The population of current cross-sectional study was selected through a multi-stage cluster sampling method. Data were collected through a questionnaire. We entered the data of 370 individuals into SPSS software (version 23, SPSS Inc., Chicago, IL, USA) and analyzed them using descriptive statistics and Mann-Whitney $U$ test. The significant level was considered as 0.05 .

Results: The mean age of the subjects was $16.29 \pm 0.89$ years. In addition, the mean menarche age and BMI were $12.82 \pm 1.09$ years and $20.67 \pm 4.17$. The incidence rates of hypomenorrhea, hypermenorrhea, amenorrhea, oligomenorrhea, and polymenorrhea were $24.6 \%, 8.4 \%, 9.7 \%, 5.7 \%$, and $13.0 \%$, respectively. There was a significant relationship between age trend with disorders like amenorrhea, oligomenorrhea, and polymenorrhea. However, there was no significant relationship between age trend with hypomenorrhea and hypermenorrhea. Generally, the prevalence of menstrual disorders increased with the increase in BMI, though this relationship was not significant. Conclusion: Due to the relatively high incidence of menstrual disorders in the present study and since numerous major illnesses such as infertility originate from these disorders, we suggest that effective measures be taken to promote the culture of menstrual health among adolescent girls and correct the misconceptions of families.

Keywords: Menstruation disturbances, Polymenorrhea, Hypomenorrhea
\end{abstract}

Received: November 1, 2020, Accepted: December 26, 2020, ePublished: December 30, 2020

*Correspondence to

Koorosh Nematpour,

Infectious and Tropical

Diseases Research Center

Hormozgan University of

Medical Sciences, Bandar

Abbas, Iran.

Tel: 09172784123

Email: koorosh19971375@

gmail.com

\section{Introduction}

Maturation is a very important process among girls which usually begins with budding breasts, followed by the emergence of pubic hair, maximization of the growth rate, and finally the menarche $(1,2)$.

The age of menarche varies in different individuals. Among the factors effective in determining the menarche age, genetics, nutritional status, public health, geographical location, exposure to light, and mental status can be noted (3). Menstrual cycle disorders are very common among the adolescent girls in the first years after menarche (4). Different figures of menstrual disorder and dysfunction have been reported in various studies among the girls after the menarche age, including $38.3 \%$ (5), 40\% (6), 45\% (7), and 55\% (8).

Menstrual periods are usually of higher regularity in the 20-40 age range and of lower regularity in the ages of below 20 and above 40 years old $(9,10)$. Usually, the interval between menstruation periods is in the range of 21-35 days among the adolescents; hence, the diagnostic criterion for disorders such as oligomenorrhea and polymenorrhea is as follows: if the interval lasts more than 35 days or less than 21 days, the individual is with oligomenorrhea or polymenorrhea, respectively (11).

The most important cause of oligomenorrhea is the impairment of hormone pathways, such as the lack of ovulation. Furthermore, diseases increasing the level of androgens, such as polycystic ovarian syndrome, adrenal hyperplasia, and hyperprolactinemia increase the risk of oligomenorrhea (12). Another very common disorder is the primary amenorrhea menstrual cycle; primary amenorrhea refers to girls who did not attain spontaneous menarche at the age of 11-15 years with or without the normal development of secondary sexual traits (13).

Hormonal drugs containing forms of estrogen and progesterone are among the most important treatments for

(c) 2020 The Author(s). This is an open access article distributed under the terms of the Creative Commons Attribution License (http:// creativecommons.org/licenses/by/4.0/), which permits unrestricted use, distribution, and reproduction in any medium, provided the original work is properly cited. 
oligomenorrhea and amenorrhea (14). Hypermenorrhea is another common menstrual disorder, which is referred to bleeding more than the usual period (5-7 days) during menstruation and may be caused by pelvic and systemic illnesses; however, its exact cause is still unknown among almost $50 \%$ of women.

Hypermenorrhea has been indicated to be effective in approximately $20 \%$ of menstrual disorders and its prevalence in girls under the age of 18 years has been reported between $8.6 \%$ and $37.5 \%$ in various countries (15).

Any disorder in the menstrual cycle of women can be a worrying factor for them; therefore, careful examination is required $(16,17)$. Accordingly, the present study aimed to determine the prevalence and factors related to the menstrual disorders among adolescent school girls aged 15-18 years in Bandar Abbas, Iran.

\section{Materials and Methods Study Design and Setting}

In this observational cross-sectional and descriptiveanalytic study, a questionnaire was used to collect the data. The study was performed in autumn of 2017 on female high school students aged 15 to 18 years old in Bandar Abbas, Iran.

\section{Study Participants and Sampling}

Based on the information taken from the Education Bureau of Bandar Abbas, the total number of female high school students in the school year of 2017-2018 was 9184 people. The sample size was obtained as 368 individuals using the Cochran's formula with a $95 \%$ confidence level and a $5 \%$ error rate; hence, 400 individuals were chosen considering drops. Sampling was performed in a multistage clustering method with the proportional to size technique, as several schools were selected from all the 61 schools in the city. Next, 10 classes were selected from among them, and 40 individuals were chosen from each class on average. All students the age range of 15-18 years old and willing to participate in the study were included. Exclusion criteria included history of taking hormonal drugs, underlying diseases such as diabetes, people with various cancers, and congenital diseases.

\section{Data Collection, Variables, and Measurements}

Data collection was performed through a researchermade questionnaire and its validity (scientific-content validity) was confirmed by several gynecologists and the drawbacks were resolved. Moreover, the reliability of the questionnaire was measured using the Cronbach's alpha and a pilot study. The first part of the questionnaire included demographic information, including age, height, weight, marital status, exercise rate, family income, and education level of parents; and the second part included questions regarding the common menstrual disorders such as amenorrhea, polymenorrhea, oligomenorrhea, etc.

Menstrual disorders were defined according to the following criteria:

- Primary amenorrhea: participants who did not attain spontaneous menarche at the age of 11-15 years with or without the normal development of secondary sexual traits (13).

- Oligomenorrhea: infrequent and irregular menstrual periods (more than 35 days without menstruation).

- Polymenorrhea: the occurrence of menstrual cycles at frequency that is higher than normal (less than 21 days).

- Hypomenorrhea: less blood flow or the duration of menses less than two days.

- Hypermenorrhea: prolonged menstrual bleeding at regular intervals (more than 8 days) (11).

In addition, this part of the questionnaire included questions about the history of taking hormonal drugs, the presence or absence of underlying diseases such as diabetes, thyroid disorders, liver disease and kidney disease. Prior to submitting the questionnaires, the research team explained the overall nature of the project and its objectives to the students and described the benefits and disadvantages of the plan to them. After completing the questionnaires, some individuals were excluded from the study due to numerous deficiencies. Finally, the data of 370 individuals entered into the SPSS software (version 23, SPSS Inc., Chicago, IL, USA) and analyzed using descriptive statistics and Mann-Whitney U test.

\section{Data Analysis}

After importing the data into the SPSS software, first, the normal or abnormal distribution of the quantitative variables such as age, body mass index (BMI), age of menarche, etc. was examined using the KolmogorovSimonov test, and the results revealed the abnormality of data of these variables. In the next step, the data examined by descriptive statistics, including mean and standard deviation (SD) of quantitative variables and frequency percentage of qualitative variables, and linearby-linear association tests and Mann-Whitney U tests. The significant level was considered as 0.05.

\section{Results}

This study was performed on 370 participants aged 1518 years old in Bandar Abbas high schools in the school year 2017-2018. The mean age of the subjects was 16.29 \pm 0.89 years with the age range of $15-18$ years. The mean menarche age was $12.82 \pm 1.09$ years, and the age range of the menarche of $61.1 \%$ of the individuals was $13-15$ years (Table 1). The mean BMI was $20.67 \pm 4.17$, with a range from 12.73 to 38.46 . The BMI was divided into three groups of low, normal, and high with values less than 19, 19-25, and more than 25, respectively. The information based on this division has been presented in Table 1. In general, $67 \%$ and $33 \%$ of the girls had normal and abnormal 
bleeding during their menstruation period, respectively. The abnormal bleeding included hypomenorrhea (24.6\%) and hypermenorrhea (8.4\%). The incidence of other menstrual disorders has been shown in Table 2.

As Figure 1 shows, the mean age of individuals with amenorrhea, oligomenorrhea, and hypomenorrhea is higher compared to that of the healthy ones; however, there is an opposite relationship for polymenorrhea and hypermenorrhea disorders, i.e., the mean age among healthy individuals is higher than that of the affected ones. The significance or insignificance of the relationship between each of these disorders and the mean age can be observed in Table 3.

Generally, the prevalence of menstrual disorders was higher with the increase in BMI; however, this trend was not significantly correlated with any of menstrual disorders (Table 3). The mean BMI for individuals with and without amenorrhea was $20.84 \pm 4.14$ and $20.65 \pm$ 4.18 , respectively; however, no significant relationship was observed $(P=0.815$, effect size $=0.852)$. Given the high rate of the effect size, it is suggested that this relationship be repeated in a study with a larger volume.

Table 1. Frequency Distribution of Students Under Study Based on Age of Menarche and BMI

\begin{tabular}{llcc}
\hline Personal Characteristics & Groups & No. & $\%$ \\
\hline \multirow{2}{*}{ Age of menarche } & $10-12$ & 141 & 38.1 \\
& $13-15$ & 226 & 61.1 \\
& $\leq 16$ & 3 & 0.8 \\
\hline \multirow{2}{*}{ BMI } & Low $(<19)$ & 152 & 41.1 \\
& Normal (19-25) & 163 & 44.1 \\
& High $(>25)$ & 54 & 14.6 \\
\hline
\end{tabular}

Abbreviation: BMI, body mass index.

Table 2. Incidence of Menstrual Disorders

\begin{tabular}{lcc}
\hline Disorders & No. & $\%$ \\
\hline Amenorrhea & 36 & 9.7 \\
\hline Oligomenorrhea & 21 & 5.7 \\
\hline Polymenorrhea & 48 & 13.0 \\
\hline Hypomenorrhea & 91 & 24.6 \\
\hline Hypermenorrhea & 31 & 8.4 \\
\hline
\end{tabular}

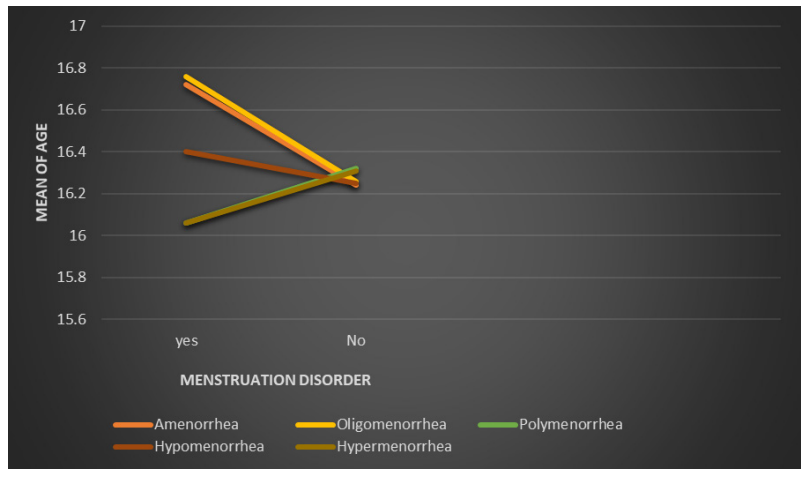

Figure 1. Relationship Between Age and the Presence or Absence of Menstrual Disorders.

As Table 3 shows, the menarche age had a significant relationship only with amenorrhea among the menstrual disorders, as the prevalence of amenorrhea was increased with the increase in the menarche age. Moreover, the mean age of the menarche was $12.85 \pm 1.11$ and $12.73 \pm 0.99$ years among the individuals with regular and irregular menstrual cycles, respectively; however, no significant relationship was observed $(P=0.340)$.

In this study, the individuals who studied articles on menstrual disorders were significantly less likely to have menstrual disorders; for example, as reported in Table 4, the incidence rate of amenorrhea was significantly lower among those who studied articles on menstrual disorders, and there was a significant relationship $(P=0.008)$.

In this study, $41 \%$ of the individuals used herbal medicines to relieve pain and improve their mental condition. In addition, none of the menstrual disorders were associated with factors, such as exercise, marital status, family income, and parental educational level.

\section{Discussion}

The mean age of the menarche in this study was 12.82 \pm 1.09 years, which is similar to the studies by Goyal et al (18) and Al-Sahab et al (19) that reported the mean menarche ages as 12.36 and 12.72, respectively. However, the mean menarche age in our study was lower than that of the studies by Naseh et al $(3.8 \pm 1.6)(20)$ and Soltani et al (3) (13.7 \pm 3.1$)$. The lower age of menarche in the present study may be attributed to the climate conditions, as well as the improved quality of nutrition and life in

Table 3. Relationship Between Menstrual Disorders with Variables of Age, Age of Menarche, and BMI

\begin{tabular}{|c|c|c|c|c|c|c|c|c|c|c|}
\hline \multirow{2}{*}{ Variable } & \multicolumn{2}{|c|}{ Amenorrhea } & \multicolumn{2}{|c|}{ Oligomenorrhea } & \multicolumn{2}{|c|}{ Polymenorrhea } & \multicolumn{2}{|c|}{ Hypomenorrhea } & \multicolumn{2}{|c|}{ Hypermenorrhea } \\
\hline & Yes & No & Yes & No & Yes & No & Yes & No & Yes & No \\
\hline Age & $16.72 \pm 0.77$ & $16.24 \pm 0.89$ & $16.76 \pm 0.62$ & $16.26 \pm 0.89$ & $16.06 \pm 0.86$ & $16.32 \pm 0.89$ & $16.40 \pm 0.88$ & $16.25 \pm 0.89$ & $16.06 \pm 0.89$ & $16.31 \pm 0.88$ \\
\hline Menarche & $14.22 \pm 1.07$ & $12.67 \pm 0.987$ & $12.52 \pm 0.92$ & $12.84 \pm 1.10$ & $12.83 \pm 1.01$ & $12.82 \pm 1.10$ & $13.05 \pm 1.14$ & $12.75 \pm 1.06$ & $12.74 \pm 1.03$ & $12.83 \pm 1.10$ \\
\hline BMI & $20.84 \pm 4.14$ & $20.65 \pm 4.18$ & $22.32 \pm 5.28$ & $20.57 \pm 4.08$ & $21.15 \pm 4.49$ & $20.60 \pm 4.13$ & $20.89 \pm 3.90$ & $20.60 \pm 4.26$ & $19.69 \pm 4.46$ & $20.76 \pm 4.14$ \\
\hline$P$ value & $\begin{array}{r}\text { For age } \\
\text { For Mena } \\
\text { For BN }\end{array}$ & $\begin{array}{l}0.002 \\
\text { rche: } 0.000 \\
\text { 1l:0.815 }\end{array}$ & $\begin{array}{r}\text { For ag } \\
\text { For mena } \\
\text { For } B N\end{array}$ & $\begin{array}{l}0.009 \\
\text { che:0.147 } \\
\text { I:0.114 }\end{array}$ & $\begin{array}{r}\text { For ag } \\
\text { For mena } \\
\text { For } \mathrm{BN}\end{array}$ & $\begin{array}{l}0.043 \\
\text { che: } 0.925 \\
\text { II:0.465 }\end{array}$ & $\begin{array}{c}\text { For ag } \\
\text { For mena } \\
\text { For } B N\end{array}$ & $\begin{array}{l}\text { e: } 0.18 \\
\text { che:0.061 } \\
\text { II:0.252 }\end{array}$ & $\begin{array}{r}\text { For ag } \\
\text { For mena } \\
\text { For } B M\end{array}$ & $\begin{array}{l}\text { e:0.09 } \\
\text { che: } 0.687 \\
\text { I: } 0.076\end{array}$ \\
\hline
\end{tabular}

Abbreviation: BMI, body mass index. 
Table 4. Relationship Between Studying Books on Menstrual Disorders with the Incidence of Amenorrhea

\begin{tabular}{|c|c|c|c|c|}
\hline \multirow{2}{*}{ Study Level } & & \multicolumn{2}{|c|}{ Amenorrhea } & \multirow{2}{*}{ Total } \\
\hline & & Yes & No & \\
\hline \multirow{2}{*}{ Very low } & Number & 23 & 136 & 159 \\
\hline & Percent & 63.9 & 40.7 & 43.0 \\
\hline \multirow{2}{*}{ Low } & Number & 12 & 130 & 142 \\
\hline & Percent & 33.3 & 38.9 & 38.4 \\
\hline \multirow{2}{*}{ Moderate } & Number & 0 & 51 & 51 \\
\hline & Percent & 0.0 & 15.3 & 13.8 \\
\hline \multirow{2}{*}{ High } & Number & 0 & 13 & 13 \\
\hline & Percent & 0.0 & 3.9 & 3.5 \\
\hline \multirow{2}{*}{ Very high } & Number & 1 & 4 & 5 \\
\hline & Percent & 2.8 & 1.2 & 1.4 \\
\hline \multirow{2}{*}{ Total } & Number & 36 & 334 & 370 \\
\hline & Percent & 100.0 & 100.0 & 100.0 \\
\hline$P$-value & & & 0.008 & \\
\hline
\end{tabular}

recent years (3).

Although the prevalence of oligomenorrhea was $5.7 \%$ in the present study, its prevalence in the studies conducted by Fathizadeh et al (21) in Babol, Iran, and Zeynalzadeh et al (22) in Tehran, Iran, was $24.6 \%$ and $25.4 \%$, respectively, which is very different from that in the present study. This difference may be attributed to such factors as different definitions of oligomenorrhea, different sample sizes, and the year in which these studies were conducted. In the studies performed by Shin et al (23) on adolescent and single girls in Korea and Naseh et al (20) on students of Birjand University of Medical Sciences in Iran, the incidence rate of oligomenorrhea was $8 \%$ and $6.3 \%$, respectively, which are close to the results obtained in the present study. In addition, as Table 2 shows, 33\% of the subjects had an abnormal bleeding time (24.6\% for hypomenorrhea and $8.4 \%$ for hypermenorrhea); this prevalence is higher than that of Soltani et al (3) with $17 \%$ and Naseh et al (20) with $15.4 \%$. Among the two disorders associated with the duration of bleeding, hypermenorrhea is a major health concern for several reasons because it is the most important cause of anemia among the girls during menstruation, and it has a profound side effect on quality of life, presence at the school, and the academic performance of the girls. Some studies have shown that hypermenorrhea is one of the most common menstrual disorders among the school and university students, and it is also the most commonly reported disorder that is not recognized or well treated (15).

The prevalence of amenorrhea was $9.7 \%$ in the present study, which was similar to the study by Rafique et al (24) with a prevalence of $9.2 \%$. This prevalence was less than that of Soltani et al (3) with a rate of $15.2 \%$; however, in the study by Bakhashandenosrat et al (25) on women with an age range of 18-40 years, the prevalence of amenorrhea was $4.5 \%$. The higher incidence of amenorrhea in this study compared to the study Bakhashandenosrat et al can be attributed to the age range of the subjects.

In our study, subjects who studied books and articles about menstrual disorders were significantly less likely to have menstrual disorders (Table 4). Similarly, in the study by Naseh et al (20), which was conducted on female students at the Birjand University of Medical Sciences, $71.3 \%$ of the subjects expressed that they needed to study the related books and articles. Therefore, studying materials on menstrual disorders is recommended for these individuals.

In this study, $41 \%$ of the subjects used herbal medicines, but in the studies by Naseh et al (20) and Hillen et al (26) this rate was $31 \%$ and $5 \%$, respectively. This significant difference can be due to various reasons, including the solid position of traditional medicine in Iranian culture, as well as the availability of the plants. In this study, there was no significant difference between the prevalence of menstrual disorders among athletes and non-athletes, which was consistent with the studies by Sundgot-Borgen et al (27) and Sehati Shafaie et al (28), but not consistent with the study by Dadgostar et al (29). The limited age range of the subjects was one of the limitations of the present study.

\section{Conclusion}

Due to the relatively high incidence of menstrual disorders in the present study and since numerous major illnesses among adults, such as infertility and various types of cancers, originate from these apparently harmless disorders, developing plans for girls in this age range is emphasized and effective measures are suggested to be taken in the field of culture-building to correct the old beliefs and customs. In addition, through holding counseling classes and training courses in schools, one can greatly contribute to the health of girls in this age range, which are in fact mothers of the future generations. Certainly, having healthy mothers physically, and perhaps more importantly, scientifically and educationally, is highly effective in training children. Given the importance of infertility among women, it is suggested that a study be conducted on the relationship between infertility and menstrual disorders.

\section{Conflict of Interest Disclosure}

The authors declare that they have no conflict of interests.

\section{Acknowledgements}

The researchers would like to appreciate the Research Committee of Hormozgan University of Medical Sciences that helped with the idea and design of this study.

\section{Ethical Statement}

This study was approved by the Ethics Committee of Hormozgan University of Medical Sciences, Bandar Abbas, Iran (IR.HUMS. REC.1395.149). This study was conducted under the supervision A personal consent was obtained from all individuals and the participants were assured that their information was fully confidential and protected. 


\section{Authors' Contributions}

Study conception and design: SS, AA, KN. Data collection: ZM, AT. Data analysis and interpretation: KN. Critical discussion and manuscript revision: AA, KN, SS. All authors read and approved the final manuscript.

\section{Funding/Support}

This study was funded by the Research Committee of Hormozgan University of Medical Sciences, Bandar Abbas, Iran.

\section{Informed Consent}

An informed consent was obtained from all participants.

\section{References}

1. Sharifi N, Najar S, Tadayon Najafabadi M, Haghighizadeh $\mathrm{MH}$. Relationship between some anthropometric indices with menarche age of girls in Ahvaz city. Journal of Ilam University of Medical Sciences. 2014;22(2):50-6. [Persian].

2. Walia DK, Yadav RJ, Pandey A, Bakshi RK. Menstrual patterns among school going adolescent girls in Chandigarh and Rural areas of Himachal Pradesh, north India. Natl J Community Med. 2015;6(4):583-6.

3. Soltani F, Shobeiri F. Menstrual patterns and its disorders in high school girls. Iran J Obstet Gynecol Infertil. 2011;14(1):2833. doi: 10.22038/ijogi.2011.5791.

4. Bieniasz J, Zak T, Laskowska-Zietek A, Noczyńska A. Przyczyny zaburzeń miesiaczkowania u nastolatek--badanie retrospektywne [Causes of menstrual disorders in adolescent girls--a retrospective study]. Endokrynol Diabetol Chor Przemiany Materii Wieku Rozw. 2006;12(3):205-10.

5. McEvoy M, Chang J, Coupey SM. Common menstrual disorders in adolescence: nursing interventions. MCN Am J Matern Child Nurs. 2004;29(1):41-9. doi: 10.1097/00005721200401000-00011.

6. Hickey M, Balen A. Menstrual disorders in adolescence: investigation and management. Hum Reprod Update. 2003;9(5):493-504. doi: 10.1093/humupd/dmg038.

7. Doi SA, Al-Zaid M, Towers PA, Scott CJ, Al-Shoumer KA. Irregular cycles and steroid hormones in polycystic ovary syndrome. Hum Reprod. 2005;20(9):2402-8. doi: 10.1093/ humrep/dei093.

8. Dzhorbenadze MT, Kristesashvili DI, Chopikashvili NA. [The frequency and character of clinical manifestations of androgenization among adolescent girls]. Georgian Med News. 2005(129):23-5.

9. Lee CA, Kadir RA, Kouides P. Inherited Bleeding Disorders in Women. John Wiley \& Sons; 2009.

10. Dasharathy SS, Mumford SL, Pollack AZ, Perkins NJ, Mattison DR, Wactawski-Wende J, et al. Menstrual bleeding patterns among regularly menstruating women. Am J Epidemiol. 2012;175(6):536-45. doi: 10.1093/aje/kwr356.

11. Omani Samani R, Almasi Hashiani A, Razavi M, Vesali S, Rezaeinejad M, Maroufizadeh $S$, et al. The prevalence of menstrual disorders in Iran: a systematic review and metaanalysis. Int J Reprod Biomed. 2018;16(11):665-78.

12. Zeinal Zadeh M, Javadian M, Hedayati F, Haji Ahmadi MJjoBUoMS. Oligomenorrhea in babol high school girls in 2001-02. 2003;5(4):57-60.

13. Balwan WK, Balwan WK, Saba N, Jamwal KS. Cytogenetic investigation of cases with primary amenorrhea in Jammu Region of Jammu and Kashmir. Int J Zool Res. 2020;10(2):1-8.

14. Deligeoroglou E, Athanasopoulos N, Tsimaris P, Dimopoulos KD, Vrachnis N, Creatsas G. Evaluation and management of adolescent amenorrhea. Ann NY Acad Sci. 2010;1205:23-32. doi: 10.1111/j.1749-6632.2010.05669.x

15. Gursel T, Biri A, Kaya Z, Sivaslıoglu S, Albayrak M. The frequency of menorrhagia and bleeding disorders in university students. Pediatr Hematol Oncol. 2014;31(5):467-74. doi: 10.3109/08880018.2014.886316.

16. Sultana A, Nadir S. Pituitary gonadotropic hormones in women with oligo/amenorrhoea. J Ayub Med Coll Abbottabad. 2008;20(3):62-5.

17. Karout N, Hawai SM, Altuwaijri S. Prevalence and pattern of menstrual disorders among Lebanese nursing students. East Mediterr Health J. 2012;18(4):346-52. doi: 10.26719/2012.18.4.346

18. Goyal P, Singh Z, Sethi GK. A cross sectional study to determine menarcheal age of adolescent bania girls from Punjab. Int J Med Sci Public Health. 2017;6(1):129-32. doi: 10.5455/ijmsph.2017.13072016572.

19. Al-Sahab B, Ardern Cl, Hamadeh MJ, Tamim H. Age at menarche in Canada: results from the National Longitudinal Survey of Children \& Youth. BMC Public Health. 2010;10:736. doi: 10.1186/1471-2458-10-736.

20. Naseh N, Hedayati $H$, Ataei $M$, Jahani N. The prevalence of menstrual disturbances in Birjand University of Medical Sciences students. Mod Care J. 2008;5(1-4):32-9. [Persian].

21. Fathizadeh N, Faraji L, Khodakarami N, Nahidi F. Menstrual disorders in early puberty in girls of 14-17 years old. J Shahrekord Univ Med Sci. 2001;3(1):41-6. [Persian].

22. Zeynalzadeh M, Javadian M, Haji Ahmadi M, Hedayati F. Oligomenorrhea in Babol high school girls in 2001-2002. J Babol Univ Med Sci. 2003;5(4):57-60. [Persian].

23. Shin SY, Lee YY, Yang SY, Yoon BK, Bae D, Choi D. Characteristics of menstruation-related problems for adolescents and premarital women in Korea. Eur J Obstet Gynecol Reprod Biol. 2005;121(2):236-42. doi: 10.1016/j. ejogrb.2004.12.017.

24. Rafique N, Al-Sheikh MH. Prevalence of menstrual problems and their association with psychological stress in young female students studying health sciences. Saudi Med J. 2018;39(1):67-73. doi: 10.15537/smj.2018.1.21438.

25. Bakhashandenosrat S, Mohammadian F, Ghaemi E, Borghei A, Joshaghani HR. Correlation of hormonal disorders with menstrual disorders. J Gorgan Univ Med Sci. 2011;13(2):8692. [Persian].

26. Hillen TI, Grbavac SL, Johnston PJ, Straton JA, Keogh JM. Primary dysmenorrhea in young Western Australian women: prevalence, impact, and knowledge of treatment. J Adolesc Health. 1999;25(1):40-5. doi: 10.1016/s1054139x(98)00147-5.

27. Sundgot-Borgen J, Torstveit MK. The female football player, disordered eating, menstrual function and bone health. Br J Sports Med. 2007;41(Suppl 1):i68-72. doi: 10.1136/ bjsm.2007.038018.

28. Sehati Shafaie F, Matin Homaei H, Zoodfekr L. Comparison the frequency of menstrual disorders (amenorrhea, oligomenorrhea, dysmenorrhea and premenstrual syndrome) between athletes and non-athletes female students of Tabriz universities, Tabriz, Iran. Iran J Obstet Gynecol Infertil. 2013;16(51):14-21. doi: 10.22038/ijogi.2013.844. [Persian].

29. Dadgostar H, Razi M, Aleyasin A, Alenabi T, Dahaghin S. The relation between athletic sports and prevalence of amenorrhea and oligomenorrhea in Iranian female athletes. Sports Med Arthrosc Rehabil Ther Technol. 2009;1(1):16. doi: 10.1186/1758-2555-1-16. 